\title{
Intravitreal ranibizumab for diabetic macular edema treatment in phakic vitrectomized eyes
}

\begin{abstract}
Purpose: To show the effect of intravitreal ranibizumab injections in treating DME in phakic vitrectomized eyes versus pseudophakic vitrectomized eyes.

Method: The medical records of 12 patients seen in the Retina Clinic and diagnosed to have DME were prospectively reviewed. The inclusion criteria were Phakic vitrectomized eyes. The exclusion criteria were pseudophkia and vitrectomized eyes with internal limiting membrane peeling. The main outcome measures included mean change in best corrected visual acuity (BCVA) from baseline, mean change in CMT, and mean number of $0.5 \mathrm{mg}$ intravitreal ranibizumab injections administered during the follow up.
\end{abstract}

Result: At baseline assessment, the mean $( \pm \mathrm{SD})$ Logarithm of the Minimum Angle of Resolution (Log MAR visual acuity) was $0.71( \pm 0.56)$. At the 12 weeks, follow up assessment the mean Log MAR VA was significantly improved to $0.32( \pm 0.20)$, $(\mathrm{p}=0.036)$. At baseline assessment, OCT was $390.75( \pm 51.40)$. The mean OCT at 4 weeks was significantly reduced to $353.83( \pm 49.49),(p=0.019)$. Furthermore, at the 8 weeks follow up assessment, the mean OCT continued its significant trend of improvement to reach $341.08( \pm 60.24),(\mathrm{p}=0.016)$ and $294.42( \pm 57.27)$ in the 8 and 12 weeks follow up assessments respectively.

Conclusion: In this small, prospective series, there was improvement in BCVA and CMT in the short-term after intravitreal Ranibizumab (IVR) for DME in previously phakic vitrectomized eyes. This may be attributable to a more remaining peripheral vitreous in phakic eyes behind the crystalline lens after vitrectomy which may clog the zonular area between anterior and posterior chambers and slowing down the antiVEGF clearance through the anterior chamber. Thus, vitreous works as a reservoir for anti-VEGF in phakic vitrectomized eyes comparing to pseudophakic vitrectomized eyes.

Keywords: ranibizumab, phakic, pseudophakic, vitrectomized, DME
Volume I Issue 4 - 2014

\author{
Ali M Al Halafi,' Ahmed Mousa² \\ 'Department of Surgery, Ophthalmology Division, Security \\ Forces Hospital, Saudi Arabia \\ ${ }^{2}$ Department of Ophthalmology, King Saud University, Saudi \\ Arabia \\ Correspondence: Ali M Al Halafi, Department of Surgery, \\ Ophthalmology Division, Security Forces Hospital, Riyadh \\ I I48I, PO Box 3643, Saudi Arabia, Tel +966- I-8024444, \\ Emailamm-ry@hotmail.com
}

Received: November 24, 2014 | Published: December I, 2014
Abbreviations: BCVA, best corrected visual acuity; IVR, intravitreal ranibizumab; DME, diabetic macular edema; VEGF-A, vascular endothelial growth factor-A; OCT, optical coherence tomography; SD-OCT, spectral domain-optical coherence tomography; CMT, central macular thickness; PPV, pars plana vitrectomy

\section{Introduction}

Diabetic retinopathy is one of the major complications of diabetes and is a leading cause of vision impairment and blindness. Approximately $75 \%$ of persons suffering from type 1 diabetes develop retinopathy, while approximately $50 \%$ of persons with type 2 diabetes may develop retinopathy, and approximately $25 \%$ of persons with diabetes may develop macular edema. ${ }^{1}$ Diabetic macular edema (DME) is the main reason for vision impairment in diabetic patients. $^{2-4}$ Vascular endothelial growth factor-A (VEGF-A) has become a frequent target for the treatment of ocular diseases in which angiogenesis plays a role as a result of the growing body of evidence that it is an important regulator of angiogenesis, vascular development and differentiation, and vascular permeability. Several anti-VEGF therapies have become available for clinical use. Ranibizumab (Lucentis, Genentech, South San Francisco, CA; Novartis Pharma, Basel, Switzerland) is a recombinant, humanized, monoclonal antibody Fab fragment against vascular endothelial growth factor A (VEGF-A), which was developed specifically for intraocular use. Intraocular pharmacokinetic data derived from studies in monkeys demonstrated that intravitreal doses of ranibizumab $(500$ or $2000 \mu \mathrm{g} /$ eye) distributed rapidly to the retina and had a vitreous half-life of 2.64.0 days. ${ }^{5}$ Past animal studies and some supportive clinical evidence, drug clearance has been generally assumed to increase and clinical drug effectiveness decrease, in vitrectomized eyes. ${ }^{6-10}$ The aim of this study to explore the result of the visual outcome and central macular thickness of diabetic macular edema treated with intravitreal ranibizumab in phakic vitrectomized eyes.

\section{Method}

The medical records of patients seen in the Retina Clinics of The Security Forces Hospital, Riyadh, Saudi Arabia, were prospectively examined. The inclusion criteria were Phakic vitrectomized eyes and diagnosed to have DME. The exclusion criteria were pseudophkia and vitrectomized eyes with internal limiting membrane peeling. Charts for demographic data including age and gender, best-corrected visual acuity, clinical findings by slit-lamp examination and fundus examination were examined. The diagnosis of DME had been confirmed by clinical fundus exam and with Optical Coherence Tomography (OCT). Retina was investigated in all patients with 
commercially available high resolution OCT (Optos OCT SLO, United Kingdom) and evaluated after pupillary dilation Spectral domain OCT (SD-OCT) was performed at each visit baseline, $4 \mathrm{wk}$, $8 \mathrm{wk}$, and $12 \mathrm{wk}$. All patients received $0.05 \mathrm{ml}$ intravitreal injections of $0.5 \mathrm{mg}$ ranibizumab administered at baseline visit then PRN at $4 \mathrm{wk}, 8 \mathrm{wk}$, and $12 \mathrm{wk}$ according to central macular thickness (CMT) when (OCT equal or more than 300 micron. Sterile surgical technique was applied for each intravitreal injection. After topical xylocaine gel has applied for 10 minutes, eyelid and eyelashes and the periocular skin were treated with $10 \%$ povidone iodine swabs, and $5 \%$ povidone iodine ophthalmic solution was applied to the conjunctival surface. Following intravitreal injection, finger-counting testing was performed. Patients self-administered topical antibiotics four times daily for 4 days after treatment. The main outcome measures included mean change in best corrected visual acuity (BCVA) from baseline, mean change in CMT, mean number of $0.5 \mathrm{mg}$ intravitreal ranibizumab injections administered during the follow up.

\section{Statistical analysis}

Data were collected from the medical records of recruited subjects and stored in a spreadsheet using Microsoft Excel 2010® software. Data management and coding were then done in excel. Data were analyzed via SPSS ${ }^{\circledR}$ version 20.0 (IBM Inc. Chicago, Illinois). Descriptive analysis was primarily done, where categorical variables were presented in the form of frequencies and percentages and continuous variables in the form of mean $( \pm \mathrm{SD})$. Inferential analysis was done in forms of comparison of pre and post intervention means using Wilcoxon Signed Ranks test. Comparison of proportion test was conducted to assess the change in percentage of cases that needed injection ( $\mathrm{T}$ test of one sample) using StatPac. Version 15.1.0, StatPac Inc. Bloomington, MN, USA. A confidence interval level was set to $95 \%$ where a corresponding $p$ value threshold was identified as 0.05 where any output $\mathrm{p}$ below 0.05 would be interpreted as an indicator of statistical significance.

\section{Results}

A total number of 12 eyes of 12 patients were included in the current study where $9(75 \%)$ were male and $3(25 \%)$ were female in the mean $( \pm \mathrm{SD})$ age of $54.7( \pm 9.4)$, range (39-68) years. The indications for pars plana vitrectomy (PPV) before DME treatment were diabetic vitreous hemorrhage in $10(80 \%)$ eyes and epiretinal membranes in $2(20 \%)$ eyes.

\section{Visual outcomes}

At baseline assessment, the mean $( \pm \mathrm{SD})$ Logarithm of the Minimum Angle of Resolution (Log MAR visual acuity) was 0.71 $( \pm 0.56)$. After 4 weeks, post first intravitreal ranibizumab injection revealed that the mean Log MAR was slightly reduced to 0.64 $( \pm 0.47)$ however insignificantly $(\mathrm{p}=0.759)$. Furthermore, at the 8 weeks, follow up assessment, although the mean Log MAR VA was much improved to $0.55( \pm 0.38)$, it was still statistically insignificant $(\mathrm{p}=0.552)$. Eventually, at the 12 weeks, follow up assessment the mean Log MAR VA was significantly improved to $0.32( \pm 0.20)$, $(\mathrm{p}=0.036)$ (Figure 1).

\section{Central macular thickness outcomes (OCT results)}

At baseline assessment, OCT was $390.75( \pm 51.40)$. The mean OCT at 4 weeks was significantly reduced to $353.83( \pm 49.49),(p=0.019)$. Furthermore, at the 8 weeks follow up assessment, the mean OCT continued its significant trend of improvement to reach 341.08 $( \pm 60.24),(p=0.016)$ and $294.42( \pm 57.27)$ in the 8 and 12 weeks follow up assessments respectively (Figure 2).

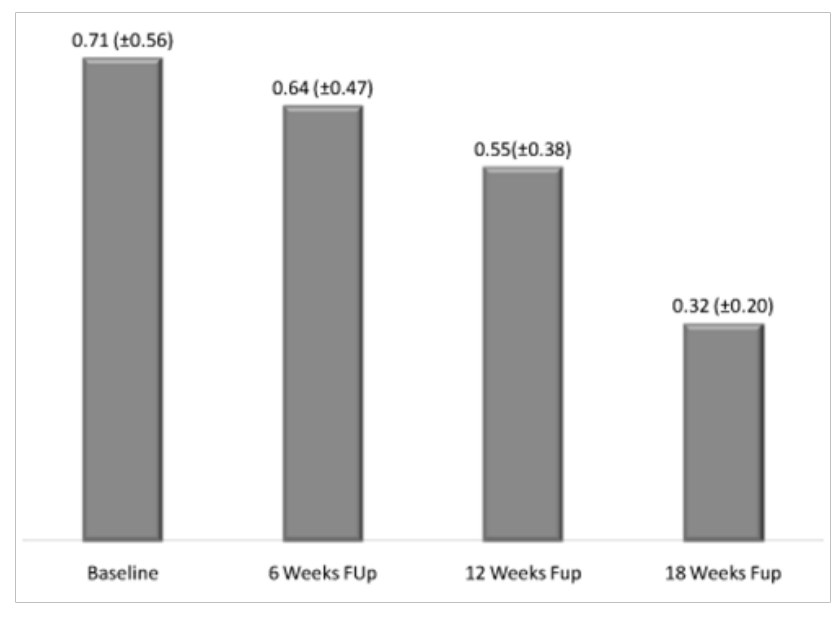

Figure I Visual acuities follow up (Fup) showed gradual vision improvement.

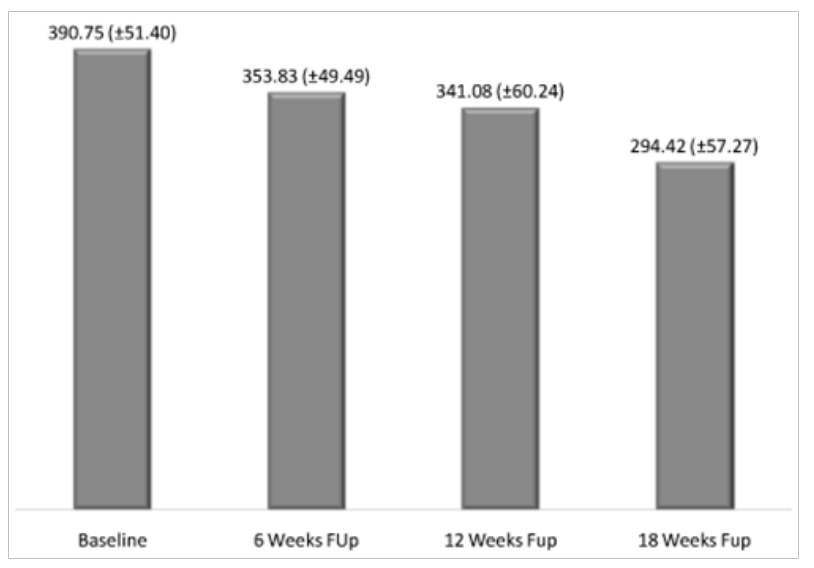

Figure 2 OCT follow up (Fup) showed gradual decrease in CMT.

\section{Frequency of ranibizumab injections}

The number of eyes in need for injection was reduced from 10 $(83.3 \%)$ in the 4 weeks follow up to $8(66.7 \%)$ in the 8 weeks follow up, however, insignificantly $(\mathrm{p}=0.645)$. This number was eventually reduced to $7(58.3 \%)$ in the last follow up assessment. Nevertheless, it also did not reach the statistical significance threshold $(\mathrm{p}=0.472)$.

\section{Discussion}

Due to the fact that studies have shown that antibiotics and steroids clear more quickly in a vitrectomized eye, retina specialists think that intravitreal injections of all drugs have a different effective between vitrectomized and non-vitrectomized eyes. In this study, we have demonstrated a significant effect of intravitreal ranibizumab injections in treating DME in phakic vitrectomized eyes. A few studies have shown a difference between Pharmacokinetics (PK) properties of intravitreal drugs in vitrectomized and those in nonvitrectomized eyes. For example, Lee et al., ${ }^{6}$ found significantly faster intravitreal elimination of the 165 amino acid splice variant of human recombinant VEGF (hVEGF165) in vitrectomized eyes (12.5 minutes) than in nonvitrectomized eyes (2.46 hours). They gave $\mathrm{VEGF}_{165}$ injections in 
rabbit eyes in vitrectomized and those in nonvitrectomized eyes and measured vitreous VEGF levels as a function of time. They found the vitreous VEGF half-life in vitrectomized eyes was 10 times shorter than that in normal eyes. Chin et al., ${ }^{7}$ showed that intravitreal triamcinolone acetonide decreases more rapidly in the vitrectomized eye than in the nonvitrectomized eye. Bakri et al., ${ }^{11}$ demonstrated that the clearance of intravitreal ranibizumab followed a mono-exponential function, with a half-life of 2.88 days. In previous studies, the halflife of intravitreal ranibizumab varied from 2.63 to 2.9 days. ${ }^{10-12}$ On the other hand, Ahn and associates ${ }^{13}$ determined the PK properties and concentrations of intravitreal ranibizumab in vitrectomized eyes are similar to nonvitrectomized rabbit eyes. They suggested that the role of the vitreous in the distribution and clearance of ranibizumab is insignificant and no difference in dosing regimens for intravitreal ranibizumab injection between vitrectomized and nonvitrectomized eyes. Increasing Oxygen transport to ischemic retinal areas as well as clearance of VEGF from these areas was shown during replacement of the vitreous gel with saline or aqueous humor which lead to reducing retinal edema and retinal neovascularisation. ${ }^{14}$ Comparing animal model studies to humans, similar differences between ocular half-life values in rabbit and monkey animal and humans have been observed for bevacizumab ${ }^{15-17}$ and triamcinolone. ${ }^{18,19}$ Krohne et al., ${ }^{20}$ quantified ranibizumab concentration in human aqueous humor samples and showed data on the intraocular PK of ranibizumab in humans. They provided an aqueous elimination half-life of ranibizumab in human eyes of 7.19 days, which is significantly longer than the respective values in animal models.

In the present study, we have demonstrated the efficacy of ranibizumab injection for patients with DME who underwent PPV in eyes without cataract surgery. Our result may suggest that ranibizumab treatment improved BCVA and decreased CMT in phakic vitrectomized eyes which may be attributable to the presence of more peripheral vitreous after core PPV in phakic eyes which leaves more vitreous reservoir for anti-VEGF therapy and less compartmental sharing between anterior and posterior chambers which may be disturbed by cataract surgery and lead to faster anti-VEGF clearance through anterior chamber. This concept may be applied by doing limited PPV instead of complete PPV in healthy vitreous associated retinal diseases such as macular holes and epiretinal membranes which may keep more vitreous for later intravitreal injections if needed. On the other hand, in pseudophakic vitrectomized eyes, there is more peripheral vitreous shaving during PPV and more compartmental sharing between anterior and posterior chambers which may lead to a faster clearance of anti-VEGF therapy. Similarly, a small, retrospective, non comparative study was done by Connor who reviewed the files of 10 patients who had undergone vitrectomy and were later on diagnosed with neovascular AMD after a mean of 5.8 years. After a mean of 5.6 injections of ranibizumab, their mean visual acuity improved from $20 / 182$ to 20/74, and their gain in Snellen lines ranged from one to five lines, with a mean of three lines. ${ }^{21}$

Another study retrospectively reviewed 11 eyes of 10 patients who underwent intravitreal bevacizumab (IVB) injections for persistent DME after a PPV that failed to resolve the edema. Those patients received monthly bevacizumab injections for three months and failed to demonstrate any change in either foveal thickness or visual acuity. ${ }^{9}$ In this study, $9(81.8 \%)$ eyes were pseudophakic and only $2(18.2$ $\%$ ) eyes were phakic eyes which may be attributable to a final result of this study. Metha et al.,22 retrospectively evaluated records of 60 eyes that underwent intravitreal bevacizumab therapy for refractory
DME. They found that vitrectomized eyes had worse visual acuity and central retinal thickness outcomes $(\mathrm{P}=.002$ and $\mathrm{P}=.028$, respectively) compared with non vitrectomized eyes. In their study, 31 (51.7\%) eyes were pseudophakic which may be attributable to the $\mathrm{p}$ value results. The frequency and the timing for IVB injection for the treatment of DME are still not clear. In the current study, the number of eyes in need for injection was reduced from $10(83.3 \%)$ in the 6 weeks, follow up to $8(66.7 \%)$ in the 12 weeks follow up. This number was eventually reduced to $7(58.3 \%)$ in the 18 weeks.

Roh et al. ${ }^{23}$ showed that improvement in visual acuity (VA) and central foveal thickness lasts for at least 6 weeks after the injections and repeated injections were required at 12 weeks for stabilization. Yuksel et al., ${ }^{24}$ reported a second IVB injection was required in 56.3 $\%$ of eyes between 6 and 9 weeks after the first injection; a third IVB injection was needed in $33.8 \%$ of eyes between 16 and 20 weeks after the second injection and a fourth IVB injection was needed in $11.3 \%$ of eyes between 25 and 29 weeks after the third injection. Harioglou et al., ${ }^{25}$ claim a second injection to $70 \%$ of patients at $6-12$ weeks after the first injection was then needed. Fang et al., ${ }^{26}$ found that improvement in VA can be maintained only for 4 weeks in the treated group and a second IVB treatment was then needed.

In this small, prospective series, there was improvement in VA and CMT in the short-term after IVR for DME in previously phakic vitrectomized eyes. This may be attributable to the remaining peripheral vitreous reservoir for anti-VEGF in phakic eyes comparing to pseudophakic eyes which decrease anterior chamber clearance. The limitations of this study were non comparative, snellen, rather than standardized ETDRS VA measurements, small sample size and relatively small follow-up. Prospective studies are required comparing the effect in vitrectomized eyes with and without cataract surgery and also different treatment regimen.

\section{Acknowledgments}

None.

\section{Conflicts of interest}

Author declares that there is no conflict of interest.

\section{References}

1. Kempen JH, O’Colmain BJ, Leske MC, et al. Prevalence of diabetic retinopathy among adults in the United States. Arch Ophthalmol. 2004;122(4):552-563.

2. Nicholson BP, Schachat AP. A review of clinical trials of anti-VEGF agents for diabetic retinopathy. Graefe's Arch Clin Exp Ophthalmol. 2010;248(7):915-930.

3. Klein R, Klein BEK, Moss SE. Visual impairment in diabetes. Ophthalmology. 1984;91(1):1-9.

4. Soheilian M, Garfami KH, Ramezani A, et al. Two-year results of a randomized trial of intravitreal bevacizumab alone or combined with triamcinolone versus laser in diabetic macular edema. Retina. 2012;32(2):314-321.

5. Gaudreault J, Fei D, Rusit J, et al. Preclinical pharmacokinetics of Ranibizumab (rhuFabV2) after a single intravitreal administration. Invest Ophthalmol Vis Sci. 2005;46(2):726-733.

6. Lee SS, Ghosn C, Yu Z, et al. Vitreous VEGF clearance is increased after vitrectomy. Invest Ophthalmol Vis Sci. 2010;51(4):2135-2138. 
7. Chin HS, Park TS, Moon YS, et al. Difference in clearance of intravitreal triamcinolone acetonide between vitrectomized and nonvitrectomized eyes. Retina. 2005;25(5):556-560.

8. Jarus G, Blumenkranz M, Hernandez E, et al. Clearance of intravitreal fluorouracil. Normal and aphakic vitrectomized eyes. Ophthalmology. 1985;92(1):91-96.

9. Yanyali A, Aytug B, Horozoglu F, et al. Bevacizumab (Avastin) for diabetic macular edema in previously vitrectomized eyes. Am J Ophthalmol. 2007;144(1):124-126

10. Kakinoki M, Sawada O, Sawada T, et al. Effect of vitrectomy on aqueous VEGF concentration and pharmacokinetics of bevacizumab in macaque monkeys. Invest Ophthalmol Vis Sci. 2012;53(9):5877-5880.

11. Bakri SJ, Snyder MR, Reid JM, et al. Pharmacokinetics of intravitreal ranibizumab (Lucentis). Ophthalmology. 2007;114(12):2179-2182.

12. Gaudreault J, Fei D, Beyer JC, et al. Pharmacokinetics and retinal distribution of ranibizumab, a humanized antibody fragment directed against VEGF-A, following intravitreal administration in rabbits. Retina. 2007;27(9):1260-1266.

13. Ahn SJ, Ahn J, Park S, et al. Intraocular pharmacokinetics of ranibizumab in vitrectomized versus nonvitrectomized eyes. Invest Ophthalmol Vis Sci. 2014;55(1):567-573.

14. Stefansson E. Physiology of vitreous surgery. Graefes Arch Clin Exp Ophthalmol. 2009;247(2):147-163.

15. Krohne TU, Eter N, Holz FG, et al. Intraocular pharmacokinetics of bevacizumab after a single intravitreal injection in humans. Am J Ophthalmol. 2008;146(4):508-512.

16. Bakri SJ, Snyder MR, Reid JM, et al. Pharmacokinetics of intravitreal bevacizumab (Avastin). Ophthalmology. 2007;114(5):855- 859.
17. Miyake T, Sawada O, Kakinoki M, et al. Pharmacokinetics of bevacizumab and its effect on vascular endothelial growth factor after intravitreal injection of bevacizumab in macaque eyes. Invest Ophthalmol Vis Sci. 2010;51(3):1606-1608.

18. Chin HS, Park TS, Moon YS, et al. Difference in clearance of intravitreal triamcinolone acetonide between vitrectomized and nonvitrectomized eyes. Retina. 2005;25(5):556-560.

19. Beer PM, Bakri SJ, Singh RJ, et al. Intraocular concentration and pharmacokinetics of triamcinolone acetonide after a single intravitreal injection. Ophthalmology. 2003;110(4):681-686.

20. Krohne TU, Liu Z, Holz FG, et al. Intraocular pharmacokinetics of ranibizumab following a single intravitreal injection in humans. Am $J$ Ophthalmol. 2012;154(4):682-686.

21. Helzner J. Lucentis in vitrectomized eyes. Retinal Physician. 2010;7(7):8.

22. Mehta S, Blinder KJ, Shah GK, et al. Intravitreal bevacizumab for the treatment of refractory diabetic macular edema. Ophthalmic Surg Lasers Imaging. 2010;41(3):323-329.

23. Roh MI, Byeon SH, Kwon OW. Repeated intravitreal injection of bevacizumab for clinically significant diabetic macular edema. Retina. 2008;28(9):1314-1318.

24. Yuksel E, Ozdek S, Yuksel N, et al. Intravitreal bevacizumab treatment for refractory diabetic macular edema. Int Ophthalmol. 2013;33(6):659-663.

25. Haritoglou C, Kook D, Neubauer A, et al. Intravitreal bevacizumab (avastin) therapy for persistent diffuse diabetic macular edema. Retina. 2006;26(9):999-1005.

26. Fang X, Sakaguchi H, Gomi F, et al. Efficacy and safety of one intravitreal injection of bevacizumab in diabetic macular oedema. Acta Ophthalmol. 2008;86(7):800-805. 